\title{
Construyendo la idea de cuadrado: Un ejemplo de la integración de GeoGebra en el currículo de $1^{\circ}$ de primaria
}

\author{
Alberto Arnal-Bailera y Belén Guerrero Belloc. Universidad de Zaragoza
}

\begin{abstract}
Recepción: 12 de mayo de 2015 | Revisión: 17 de junio de 2015 | Aceptación/Publicación: 17 de julio de 2015
Correspondecia: albarnal@unizar.es | belengbelloc@gmail.com

Citar: Arnal-Bailera, A. Y Gerrero-Belloc, B. (2015). Construyendo la idea de cuadrado: Un ejemplo de la integración de
\end{abstract} GeoGebra en el currículo de 1ㅇ de primaria. ReiDoCrea, 4, 129-135.

\begin{abstract}
Resumen: La integración de los programas informáticos de propósito didáctico es todavía incipiente en la práctica docente en los primeros cursos de Educación Primaria. No obstante, hay una gran cantidad de material accesible a través de Internet cuya adecuación, o en su caso adaptabilidad, a estos niveles merece la pena investigar. Se realiza una revisión de applets, aplicaciones realizadas con el software de geometría dinámica GeoGebra, en páginas institucionales españolas para seleccionar aquellos que puedan ser más cercanos a $1^{\circ}$ de primaria en el tratamiento de los objetos matemáticos. Presentamos aquí una de las aplicaciones seleccionadas, el análisis de la misma junto con las adaptaciones realizadas y la puesta en práctica en el aula. Los resultados obtenidos informan de las debilidades didácticas de la oferta disponible y del necesario trabajo intermedio entre la obtención de la aplicación y su utilización en el aula. GeoGebra muestra claras potencialidades en el apoyo a la construcción compartida del conocimiento matemático en esta etapa, en este caso el concepto de cuadrado.
\end{abstract}

Palabras clave: Matemáticas, Geometría plana, GeoGebra, Programa informático didáctico

BUILDING A IDEA OF A SQUARE

\begin{abstract}
The integration of learning software in school is still at an early stage in the first levels of Primary Education. However, there is a great amount of material accessible on the Internet which suitability or adaptability to these levels is worth exploring. A review of applets designed with GeoGebra (dynamic geometry software) is carried out in institutional websites to select those that are most suitable depending on the way that mathematical objects are processed. The results obtained inform about the didactic weakness of the accessible applets and the need for additional work to use them at school. GeoGebra shows clear potential supporting the shared construction of mathematical knowledge, in this case the concept of square.
\end{abstract}

Keywords: Mathematics, Plane Geometry, GeoGebra, Educational software

\section{Introducción}

Nos encontramos en un momento en la que la presencia de las nuevas tecnologías en las aulas de primaria es creciente. Desde la pedagogía ha hecho un esfuerzo en analizar las posibilidades de integración de los nuevos medios disponibles en el sistema de enseñanza de nuestro país (Cabero, 2006; García, Joaquín, Torres y Vázquez, 2013). También la didáctica de las matemáticas se ha ocupado de este asunto, aunque los esfuerzos han estado mayoritariamente dirigidos a la etapa de Secundaria con valiosas aportaciones a la didáctica de la Geometría tanto a nivel nacional (Iranzo y Fortuny, 2009) como internacional (Mariotti, 2013)

En el tópico que nos ocupa, la enseñanza de los cuadriláteros y específicamente el cuadrado, también se han hecho avances en cuanto al aprovechamiento de GeoGebra, software de creciente implantación en nuestras aulas. La literatura disponible plantea secuencias de enseñanza diseñadas a menudo por equipos mixtos formados por investigadores en didáctica de las matemáticas y por profesores en ejercicio (Arnal y Planas, 2013).

Este diseño no se ocupa de un hecho relevante en la práctica escolar como es el reaprovechamiento que muchos profesores tratan de hacer de aquellas aplicaciones 
ya realizadas. Este reaprovechamiento es un proceso que debe ser profundamente reflexionado tanto desde un punto de vista tecnológico, analizando el grado de adquisición de la herramienta o génesis instrumental (Drijvers et al., 2010; Trouche, 2004), como desde un punto de vista más didáctico y matemático, analizando el tratamiento de los objetos matemáticos en la aplicación en cuestión y las dificultades de aprendizaje que puedan aparecer.

Trabajamos entonces con los objetivos de contrastar la validez de un proceso de selección, análisis y adaptación de una actividad realizada con GeoGebra para la enseñanza y aprendizaje del concepto de cuadrado y de la relevancia de su implementación en primero de Educación Primaria.

El trabajo que presentamos aquí forma parte de una investigación más amplia en la que se estudia una secuencia didáctica completa para desarrollar la enseñanza y aprendizaje de los polígonos en primero de Educación Primaria en un Centro de Zaragoza. Por razones de espacio describiremos el proceso completo realizado con una de las nueve actividades que componen la secuencia, desde el momento en que localizamos la actividad original, pasando por el uso directo de la actividad con alumnos una vez modificada y terminando en las indicaciones para la gestión del debate que con los alumnos a posteriori que permite analizar el aprovechamiento de la secuencia.

\section{Métodos}

Con el objetivo de diseñar una secuencia de actividades para la enseñanza y aprendizaje de los cuadriláteros en $1^{\circ}$ de Primaria, se realizó una selección de entre las aplicaciones GeoGebra que algunas páginas web institucionales o de profesionales de reconocido prestigio ofertaban con acceso libre y gratuito. La página web que utilizamos con mayor frecuencia y de la que extrajimos la actividad original que nos ocupa fue el "Proyecto Gauss" del Ministerio de Educación de España.

La aplicación de la secuencia se llevó a cabo en el CEIP "Miraflores" de Zaragoza con dos grupos de alumnos y alumnas de 6 a 7 años de $1^{\circ}$ de Educación Primaria.

\section{La actividad}

En la citada página web, seleccionamos la actividad denominada "falsos cuadrados" para su análisis matemático y tecnológico y posterior modificación, (ver figura 1).
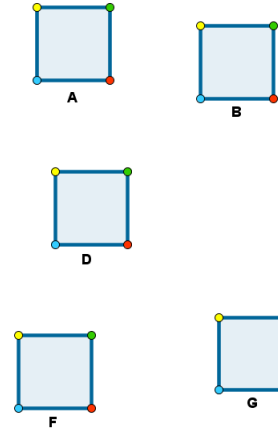
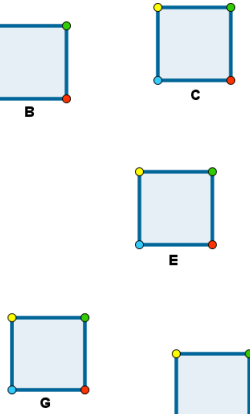
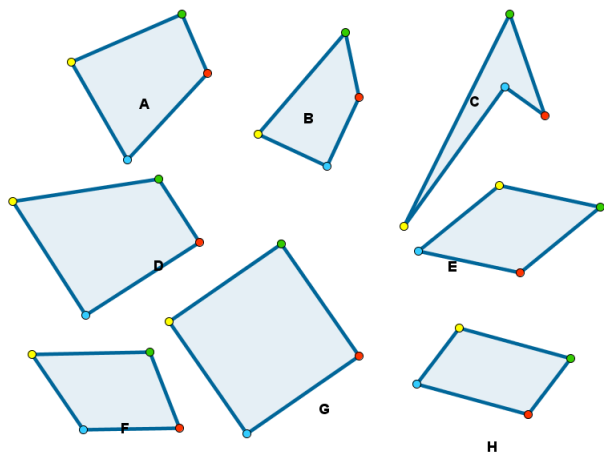

Figura 1. Actividad "falsos cuadrados" con resolución. 
Esta actividad es específica para el trabajo con cuadrados. Está clasificada por la página web para su trabajo en $5^{\circ}$ y $6^{\circ}$ de Educación Primaria.

La actividad propuesta es matemáticamente limitada, ya que ofrece muchos ejemplos de cuadrados que pierden simultáneamente la igualdad de ángulos y lados pero no ofrece ninguno que pierda solo la igualdad de lados y solo uno que pierde la igualdad de ángulos al arrastrar. Por tanto en la resolución solo aparece un rombo y ningún rectángulo. Si consideramos que la actividad es para $5^{\circ}$ y $6^{\circ}$ de Primaria, echamos de menos que se pueda, al menos optativamente, ver la medida de los ángulos, de los lados o el trazo de las diagonales.

Tecnológicamente, la actividad requiere un esfuerzo pequeño del alumno que solo tiene que realizar un test de arrastre para comprobar que los dibujos de cuadrados realmente corresponden con figuras cuadradas (Gutiérrez 2012). No obstante en algunos ejemplos hay que acertar con el vértice que deforma el cuadrado y en otros casos algunos de los vértices no son libres y, o bien no se mueven en absoluto, o bien se mueven siguiendo a otro vértice.

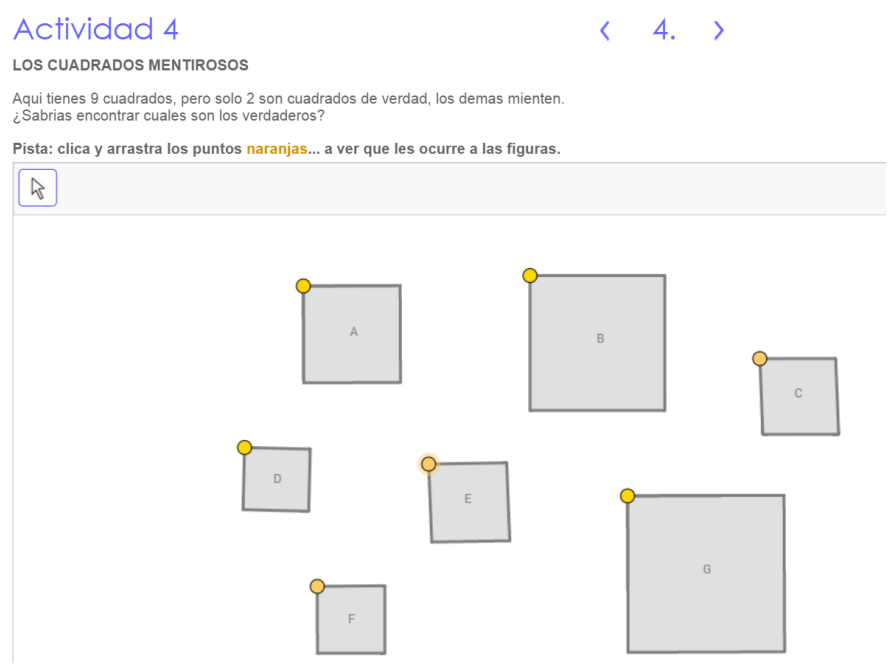

Figura 2. Portada de la actividad propuesta.

Se dan unas pequeñas indicaciones del trabajo a realizar por los alumnos "Mueve los vértices de cada cuadrado y después contesta a las preguntas" pero no se dan indicaciones del trabajo a realizar por el profesor o cómo gestionar las diversas respuestas que pueden aparecer. La segunda pregunta es ambigua en su planteamiento ya que dice "¿Por qué las otras parecían cuadrados cuando no lo eran?" que puede hacer fijarse al alumno en las figuras originales, cuando lo interesante es centrarse en qué propiedades del cuadrado se han perdido o se mantienen.

Pasamos a comentar ahora las modificaciones que, por un lado la enriquecen matemáticamente y por otro permiten un uso tecnológicamente más sencillo en primero de primaria:

La primera variación que se introdujo fue colocar cuadrados de diferentes tamaños, para evitar la creación estereotipos tempranos en los alumnos (ver figura 2). Aunque todos los cuadrados se presentan de la manera más común (con sus lados paralelos a los bordes de la pantalla o la hoja) esta no es la tarea principal, ya que colocándolos de forma girada podría haber provocado la reacción entre los alumnos de negar que 
aquello era un cuadrado por la falta de ejemplos vistos anteriormente. Esta carencia de variación en la posición de los cuadrados se solventa durante la resolución del ejercicio dado que los cuadrados verdaderos al moverlos aumentan de tamaño girando sobre uno de sus vértices. La segunda variante introducida, fue variar la manera de deformarse de los cuadrados, incluyendo esta vez los que se deformaban en rectángulos y en rombos, introduciendo dos de cada tipo (ver figura 3 ).
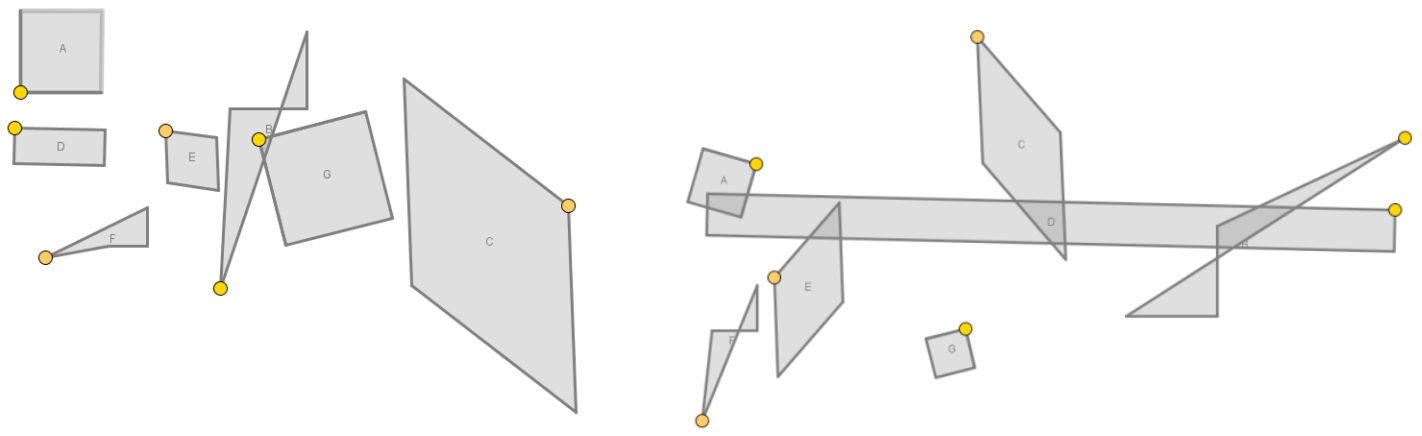

Figura 3. Ejemplos de la actividad una vez finalizada por los alumnos.

En esta actividad, las posibilidades que nos da GeoGebra son clave para desarrollar ese ejercicio, dado que en la realidad es muy difícil, por no decir imposible, reproducirla. Podemos por ejemplo construir un cuadrado con fichas y corchetes, sin embargo ese cuadrado tan solo se podrá deformar en un rombo, sin estirar los lados. Así pues, GeoGebra se convierte en este caso en contexto fundamental para desarrollar esta actividad, que a nuestro modo de ver es muy útil y curiosa ya que tendrá que hacer a los niños reflexionar sobre sus decisiones, ya que si leemos el enunciado propuesto en la imagen anterior, preguntaremos a los niños por los cuadrados "verdaderos" en un intento temprano de introducción de manera informal de la diferencia entre figura y dibujo. Gutiérrez (2012) trabaja esta diferenciación en el contexto del software de Geometría dinámica, reconociendo que una figura es un objeto geométrico abstracto caracterizado por las propiedades matemáticas derivadas, y un dibujo es su representación. No es posible saber, sin utilizar el test de arrastre, qué figura hay detrás de un dibujo que vemos en la pantalla porque es necesaria saber qué herramientas se han usado para la construcción.

La actividad modificada se puede consultar a través de Internet, al haber sido alojada en GeoGebraTube.

\section{Los instrumentos}

Los instrumentos utilizados para la toma de datos son:

- Los archivos donde se guardan las distintas resoluciones de la tarea de los alumnos.

- Las explicaciones escritas de los alumnos. Los alumnos tenían que responder a dos preguntas. Ver figura 4.

- Las anotaciones sobre los diálogos mantenidos con la profesora durante la realización de la actividad. Estos diálogos seguían un esquema semiestructurado en los que la primera pregunta era siempre la misma y la segunda y tercera dependían de la respuesta de los alumnos a la primera.

Uno de los objetivos de las actividades es provocar un diálogo posterior con la profesora en gran grupo. Para prepararlo, la profesora pasa por cada pareja tratando 
de promover la reflexión de los alumnos sobre el porqué de las respuestas que van dando. Dada la edad de los alumnos se considera que no es suficiente con preguntar por escrito y que se obtiene mayor información y reflexión mediante este instrumento oral.

Realizamos un análisis cuantitativo solo en lo relativo a la corrección matemática de las respuestas a la primera pregunta (ver figura 4) “¿Cuáles son los cuadrados verdaderos?"

\section{(4) ¿Cuáles son los cuadrados verdaderos? Escribe las letras}

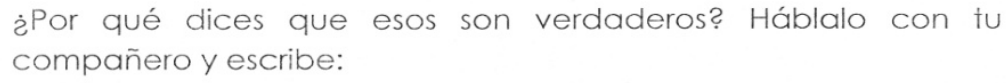

Figura 4. Preguntas escritas de la actividad.

Sobre los demás datos obtenidos, el análisis realizado es de tipo cualitativo. Las unidades de análisis son las anotaciones de los alumnos y las transcripciones de los diálogos con la profesora. Las distintas categorías se construyen a partir de una aproximación inductiva en la que el propio análisis da lugar a las categorías (Berg, 2007). La validez y fiabilidad internas se mejoran con la presencia de dos investigadores que actúan sobre los mismos registros observacionales (Hernández, Fernández y Baptista, 2010, p. 476).

\section{Resultados}

A partir de revisiones sucesivas de los datos obtenidos a través de los instrumentos, surgen dos temas sobre los que tratan los comentarios de los alumnos que se convierten en categorías de análisis que exponemos a continuación ilustrándolas con ejemplos para mayor claridad (ver tabla 1 ):

\begin{tabular}{|c|l|l|}
\hline \multicolumn{3}{|c|}{ Tabla 1. Categorías para el análisis cualitativo. } \\
\hline Dinamismo & \multicolumn{1}{|c|}{ Descripción } & \multicolumn{1}{c|}{ Ejemplo } \\
\hline & $\begin{array}{l}\text { El alumno utiliza en sus respuestas verbos } \\
\text { o gestos relacionados con movimientos. }\end{array}$ & "Se mueve como un cuadrado" \\
"No se convierte en otra figura" \\
\hline Matemáticas & $\begin{array}{l}\text { El alumno utiliza en sus respuestas alguna } \\
\text { de las características matemáticas del } \\
\text { cuadrado }\end{array}$ & "Están rectos" \\
\hline
\end{tabular}

Ante la primera pregunta de la profesora "¿Y por qué decís que esos son los cuadrados de verdad?", la mayor parte de los alumnos recurren a las características de dinamismo de la actividad y argumentan utilizando verbos como "mover", "convertir", "doblar" o "estirar" (ver figura 5). Podemos decir que las explicaciones adquieren dinamismo. La respuesta "porque se mueve como un cuadrado" fue la respuesta más frecuente en ambas clases. Podemos pensar que los niños sí que distinguen lo que es un cuadrado por su forma, la simetría de sus lados, etc. Sin embargo no saben decir porque sigue siendo un cuadrado al tirar del punto, ellos reconocen que cuando mueven el punto, la figura sigue manteniendo esa forma que ellos reconocen como cuadrado, y sin embargo los otros no lo hacen, se deforman y por tanto "no se mueven como cuadrados". 


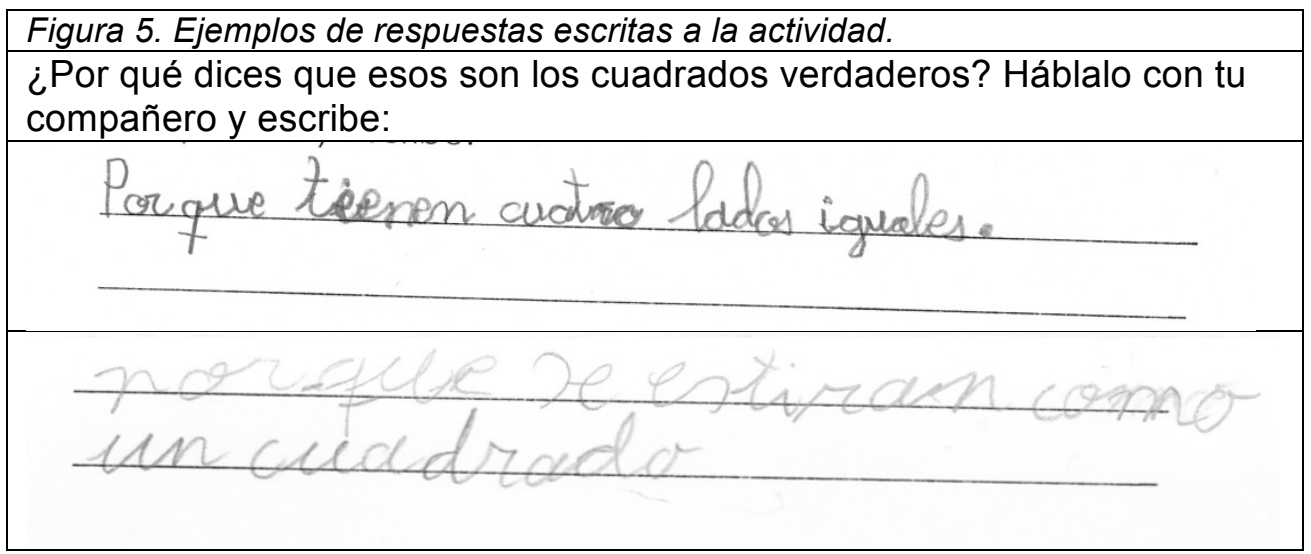

Un grupo menor de alumnos que logran relacionar una de las características matemáticas de estas figuras que, aunque no aparece aún en el libro de texto, los maestros sí que han comunicado a sus alumnos. Esta característica es la igualdad de lados, que como vemos en la figura 5, utilizan para contestarnos aunque esta respuesta no sea del todo cierta o este incompleta. Es llamativa la respuesta de una pareja "...no se convierte en otra figura. Mira este es un rombo y este es un rectángulo y este es algo raro." Muestra la capacidad de estos alumnos para clasificar cuadriláteros que apenas habían leído en el libro anteriormente.

Que emerjan estas dos categorías de análisis nos permitirá hilar la explicación posterior de la profesora, enlazando adecuadamente lo visto en el programa con las características matemáticas del cuadrado, habitualmente presentadas en forma estática. Sería deseable que los alumnos captaran las características dinámicas de la actividad, para a través del diálogo con la profesora y los compañeros, terminara adquiriendo también las características matemáticas. Esta directriz marcará el diálogo posterior donde la profesora tratará de hacer una construcción compartida del concepto de cuadrado.

Cabe señalar que todas las parejas que intervinieron en la actividad fueron capaces de realizar la tarea correctamente en cuanto a identificar los cuadrados que resultaban tras aplicar el test de arrastre. Por tanto podemos decir que los alumnos participantes no tuvieron problemas de reconocimiento de cuadrados, independientemente del tamaño u orientación que tuvieran.

Los alumnos se encontraron cómodos razonando y trabajando con la herramienta GeoGebra, sin requerir en ningún momento ningún otro instrumento como una regla u otros. Podemos afirmar que el punto de génesis instrumental de GeoGebra es adecuado para el planteamiento de esta actividad.

\section{Discusión y conclusiones}

En cuanto a la consecución del primer objetivo, "contrastar la validez de un proceso de selección, análisis y adaptación de una actividad realizada con GeoGebra para la enseñanza y aprendizaje del concepto de cuadrado", a tenor de los datos obtenidos dicho proceso ha sido realizable y es plenamente replicable para otros contenidos. Respecto al segundo objetivo, "contrastar la relevancia de su implementación en primero de Educación Primaria" podemos afirmar que las actividades han sido realizadas por la práctica totalidad de los alumnos y los resultados de las mismas han alimentado la construcción del concepto de cuadrado. 
Las respuestas de los alumnos a las preguntas formuladas fueron verdaderamente ricas y mostraron tanto las ideas de dinamismo aportadas por GeoGebra, como las ideas matemáticas que queríamos que surgieran para guiar la construcción posterior del conocimiento matemático en gran grupo. Esto muestra la potencialidad de GeoGebra como motor de la discusión matemática también en los primeros cursos de Educación Primaria, en consonancia con lo aportado por investigadores como Mariotti (2013) con alumnos de secundaria. Bajo una mirada más tecnológica, podemos constatar que, en línea con lo aportado en investigaciones anteriores (Arnal y Planas, 2013), una vez elegido el instrumento tanto alumno como, sobre todo, profesor pueden y se deben mantener fieles a él procurando elaborar respectivamente los razonamientos y las preguntas que guían el diálogo en torno al mismo.

\section{Referencias}

Arnal, A. \& Planas, N. (2013). Uso de tecnología en el aprendizaje de la Geometría con grupos de riesgo: un enfoque discursivo. En Berciano, A.; Gutiérrez, G.; Estepa, A. \& Climent, N. (Eds.), Investigación en Educación Matemática XVII (157-164). Bilbao: SEIEM.

Arnal-Bailera, A. \& Guerrero, B. Los cuadrados mentirosos. URL: https://tube.geogebra.org/student/b887119\#material/919005. Accessed: 2015-06-25. (Archived by WebCite ${ }^{\circledR}$ at http://www.webcitation.org/6ZYO4gMsn)

Berg, B.L. (2007). Qualitative research methods for the social sciences. Boston: Allyn and Bacon.

Cabero, J. (2006). Bases pedagógicas para la integración de las TIC en Primaria y Secundaria. Universidad de Sevilla: Grupo de Tecnología Educativa.

Drijvers, P., Doorman, M., Boon, P., Reed, H. \& Gravemeijer, K. (2010). The teacher and the tool: instrumental orchestrations in the technology-rich mathematics classroom. Educational Studies in Mathematics, 75(2), 213234.

García, D. M., Joaquín, M., Torres, P., \& Vázquez, I. R. (2013). Estilos de enseñanza y las nuevas tecnologías en la educación.

Gutiérrez, A., \& Jaime, A. (2012). Reflexiones sobre la enseñanza de la geometría en primaria y secundaria. Tecné, Episteme y Didaxis: TED, 32, 55-70.

Hernández, R., Fernández, C. \& Baptista, M.P. (2010) Metodología de la Investigación. México: McGraw Hill Educación.

Iranzo, N. \& Fortuny, J.M. (2009). La influencia conjunta del uso de GeoGebra y lápiz y papel en la adquisición de competencias del alumnado. Enseñanza de las Ciencias, 27(3), 433-446.

Mariotti, M. A. (2013). Introducing students to geometric theorems: how the teacher can exploit the semiotic potential of a DGS. ZDM, 45(3), 441-452.

Ministerio de Educación, Cultura y Deporte. Instituto Nacional de Tecnologías Educativas y Formación del Profesorado $\begin{array}{ll}\text { Proyecto } & \text { Gauss. 2015-06-25. }\end{array}$ URL:http://recursostic.educacion.es/gauss/web/materiales_didacticos/primaria/actividades/geometria_poligon os.htm. Accessed: 2015-06-25. (Archived by WebCite ${ }^{\circledR}$ at http://www.webcitation.org/6ZYNbosSC)

Trouche, L. (2004). Managing the complexity of human/machine interactions in computerized learning environments: guiding students' command process through instrumental orchestrations. International Journal of Computers for Mathematical Learning, 9(3), 281-307. 